\title{
Spectroscopic Detection of Gastrointestinal Dysplasia Using Optical Microsensors
}

\author{
D. S. Ferreira*, Student Member, IEEE, V. C. Pinto, J. H. Correia, Member, IEEE, \\ and G. Minas, Senior Member, IEEE
}

\begin{abstract}
The detection of dysplasia in the gastrointestinal tract can be performed using optical microsensors based on thin-film optical filters and silicon photodiodes. This paper describes two optical microsensors that can be used for spectroscopy data collection in two different spectral bands (one in the violet/blue region and the other in the green region) for which two optical filters were designed and fabricated. An empirical analysis of gastrointestinal spectroscopic data using these specific spectral bands is performed. The obtained results show that it is possible to accurately differentiate dysplastic lesions from normal tissue, with a sensitivity and specificity of $77.8 \%$ and $97.6 \%$, respectively. Therefore, the developed filters can be used as a tool to aid in diagnosis. The small size of the optical microsensors can enable, in the future, integration in endoscopic capsules.
\end{abstract}

Index Terms-Diffuse-reflectance, Fabry-Perot optical filters, fluorescence, spectroscopy tissue diagnosis.

\section{INTRODUCTION}

G ASTROINTESTINAL (GI) cancers, in particular cancers of the esophagus, stomach, and colorectum, are among the fifth leading causes of cancer-related deaths worldwide [1]. These epithelial cancers are usually preceded by dysplastic changes. An early identification of cancer is very important since the chances of an effective treatment considerably increase when the disease is detected at the dysplastic stage, improving the survival rate [2], [3]. The World Health Organization has estimated that nearly one-third of cancer burden could be decreased if lesions were diagnosed and treated at its earliest stages. Unfortunately, dysplastic lesions are difficult to detect using standard screening methods: dysplasia is not readily identifiable by visual inspection during routine endoscopy. As a consequence, physicians often take a large number of undirected

Manuscript received March 15, 2011; revised May 11, 2011; accepted June 6, 2011. Date of publication June 23, 2011; date of current version August 19, 2011. This work was supported by ADI with reference FCOMP-01-0202FEDER-005358, by FEDER funds through the "Programa Operacional Factores de Competitividade-COMPETE", and by national funds by Fundação para a Ciência e a Tecnologia, with project reference PTDC/BIO/70017/2006, and the MIT|Portugal Program (SFRH/BD/38978/2007). Asterisk indicates corresponding author.

*D. S. Ferreira is with the Centro Algoritmi, University of Minho, Campus de Azurém, 4800-058 Guimarães, Portugal (e-mail: debora@dei. uminho.pt).

V. C. Pinto, J. H. Correia, and G. Minas are with the Centro Algoritmi, University of Minho, Campus de Azurém, 4800-058 Guimarães, Portugal (e-mail: vpinto@dei.uminho.pt; higino.correia@dei.uminho.pt; gminas@dei. uminho.pt).

Color versions of one or more of the figures in this paper are available online at http://ieeexplore.ieee.org.

Digital Object Identifier 10.1109/TBME.2011.2160263 biopsies to increase the chances of detecting invisible lesions, which results in sampling errors and high costs associated with the procedure [2]-[4].

Optical techniques may overcome some limitations of current screening methods and enhance detection of dysplasia in vivo. Diffuse-reflectance and fluorescence spectroscopy have been proven to be highly sensitive for the identification of dysplasia, by exhibiting different spectral features that can be correlated with normal and cancerous tissue [2], [3]. Such techniques have the ability to reveal biochemical and morphological tissue information that can be used to characterize changes that take place during disease transformation [3], [4].

Several studies have successfully applied diffuse-reflectance and fluorescence spectroscopy for detecting and classifying dysplasia in a variety of GI structures [5]-[7]. In these studies, data collection is performed using costly and bulky clinical instruments, usually composed by a spectrograph, a detector, and optical fibers. This instrument configuration has some drawbacks: regular optical fibers might have low collection efficiency, thus requiring high quantum efficiency detectors (such as chargecoupled device (CCD) cameras), and, the detector is placed at the distal end of the collection fiber, where considerable light might be lost [8]. Moreover, catheter-based endoscopy systems are invasive and very uncomfortable for the patient. The development of a miniature spectroscopy system without using optical fibers, spectrograph or CCDs would enable integration in small and less-invasive devices for GI tract evaluation. Kfouri et al. [9] have already developed a miniaturized fluorescence imaging device for noninvasive diagnosis of diseases in the GI tract. However, the full-integrated prototype is still too large ( $2 \mathrm{~cm}$ in diameter and $10 \mathrm{~cm}$ in length), and further miniaturization is compromised by the use of several incompatible CMOS IC components. Also, only fluorescence images are acquired to perform diagnosis. To the best of our knowledge, it was not yet developed a miniaturized device that integrates both fluorescence and diffuse-reflectance functions for early cancer diagnosis.

In this study, it is described a strategy for the detection of dysplasia in the upper GI tract and proposed the development of a low complexity, miniaturized device for spectroscopy data collection (fluorescence together with diffuse-reflectance). This system, named as optical microsensor, is based on thin-film optical filters and CMOS silicon photodiodes, for the selection and detection of two different spectral bands that are significant for the identification of GI dysplastic lesions. This approach requires the development of two optical filters, one of which will be used for fluorescence measurements, while the other will be 
used to collect the reflectance signal. Both filters will have a Fabry-Perot multilayer structure made with dielectric materials that are Si compatible, thus compatible with standard microelectronics fabrication and processing techniques (which is an asset toward the development of a fully miniaturized device, in a single chip). Also, the use of all-dielectric layers allows a narrow and a high peak transmittance spectral band, as required for the application [10]-[12].

The proposed device has three major advantages over previous systems: 1) it is miniaturized and, thus, suitable for a future integration in a less-invasive medical device (e.g., endoscopic capsule); 2) it integrates components that are compatible with standard microelectronics fabrication and processing techniques; and 3) it combines two different spectroscopy techniques: diffuse-reflectance and fluorescence.

\section{DIFFUSE-REFLECTANCE AND FLUORESCENCE SPECTROSCOPY}

In diffuse-reflectance spectroscopy, white light delivered to the tissue is absorbed and multiple scattered by tissue constituents and part of it returns, carrying information about tissue optical properties (scattering and absorption) [4], [13]. Scattering is mainly originated by collagen fibers in connective tissue, whereas absorption is mainly due to hemoglobin. The major hemoglobin absorption peaks are nearly the $420 \mathrm{~nm}$ and 540$580 \mathrm{~nm}$ regions [4]. A decrease in scattering is associated with increasing dysplasia: epithelial thickness increases, and therefore the amount of light propagating to the connective tissue with collagen is reduced. Additionally, with dysplasia some enzymes are released that will cleave the collagen cross links [3], [14]. An increased hemoglobin concentration, which translates into an increased absorption, may be associated with angiogenesis that is proved to be linked with some types of early cancers [15].

Epithelial tissues produce fluorescence when excited by ultraviolet (UV) or short-wavelength visible light. This fluorescence is originated by several fluorophores that are related to the structural arrangement and metabolism of cells. Each fluorophore is excited by a specific wavelength and has particular emission characteristics, supplying different biochemical information. A modification in fluorescence emission may be related to the emergence of pathological conditions. The dominant fluorescent tissue layer is the submucosa, where collagen and elastin emit around the $400 \mathrm{~nm}$ spectral band [4], [16], [17]. NAD(P)H and collagen are considered fluorescent biomarkers of precancerous changes in epithelial tissues [14]. An increase in $\mathrm{NAD}(\mathrm{P}) \mathrm{H}$ is associated with an increase in cellular metabolic activity and proliferation, both of which occur with the progression of dysplasia. A decrease in collagen can be an indicator of loss of structural integrity and is translated by low fluorescence intensity. Thus, fluorescence intensity of dysplastic epithelial tissue is generally lower compared to healthy tissue [14], [18], [19]. This concept, together with diffuse-reflectance signal information, can be used to distinguish the presence and absence of malignancy.

An empirical analysis of fluorescence and diffuse-reflectance signal from GI tissue is described. The intensity of fluorescence

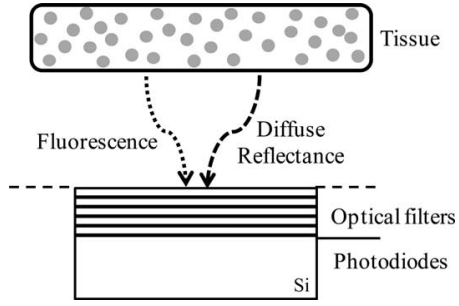

Fig. 1. Cross-sectional view of the optical microsensor with optical filters and photodiodes (not scaled).

and diffuse-reflectance within only two specific spectral bands will be used for the discrimination of dysplastic and normal tissue.

\section{Design of the Optical Microsensor}

An optical microsensor for spectroscopy data collection was designed (see Fig. 1). This device is based on thin-film optical filters and silicon photodiodes that will, respectively, select and detect several light wavelengths of interest for the diagnosis of GI dysplasia. The use of these components obviates the need for expensive and sophisticated detection equipment and optical fibers for light collection from tissues. In addition, small-size UV and white light-emitting diodes (LEDs) could be incorporated in the instrument as the illumination sources for fluorescence and diffuse-reflectance measurements, respectively.

The designed microsensor can be scaled down to a few millimeters, and like that has potential to be integrated in very small and thus less-invasive devices, such as the endoscopic capsule. For this particular application, a control of the capsule direction and distance to the tissue surface has first to be achieved. Promising developments in this field have already been made, which may allow, in the future, to place the capsule into gentle contact with the tissue, in a controlled way, to collect spectroscopy data [20], [21]. This placement of the detection system close to the sample allows us collecting most of the reemitted light.

Concerning power supply requirements, for the proposed optical microsensor, it will be similar to the one used in commercial endoscopic capsules. A 50-mAh capacity with a 3-V nominal voltage would be used, being enough for 6-h work. Also, it is important to notice that the illumination sources will not spend more energy than the one currently available since when the UV LEDs are turned ON, the white LEDs are turned OFF and vice versa.

\section{A. Optical Filters Structure and Materials}

The detection of specific spectral bands requires the use of thin-film optical filters placed on top of silicon photodiodes. The filtering system is based on Fabry-Perot thin-film optical resonators. These consist of two flat parallel mirrors separated by a specific distance, with a resonance cavity in the middle (see Fig. 2). The thickness of the resonance cavity determines the transmitted wavelength band, as explained by the equation $\lambda q$ $=2 n d$, where $n$ is the refractive index of the cavity medium, $d$ is the cavity length or thickness, $\lambda$ is the transmitted wavelength, 


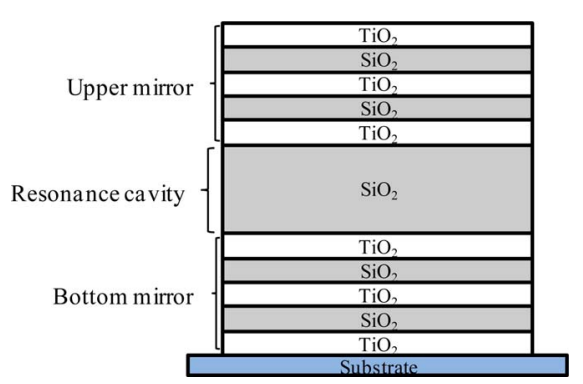

Fig. 2. Designed Fabry-Perot resonator, composed by 11 layers, and with dielectric mirrors.

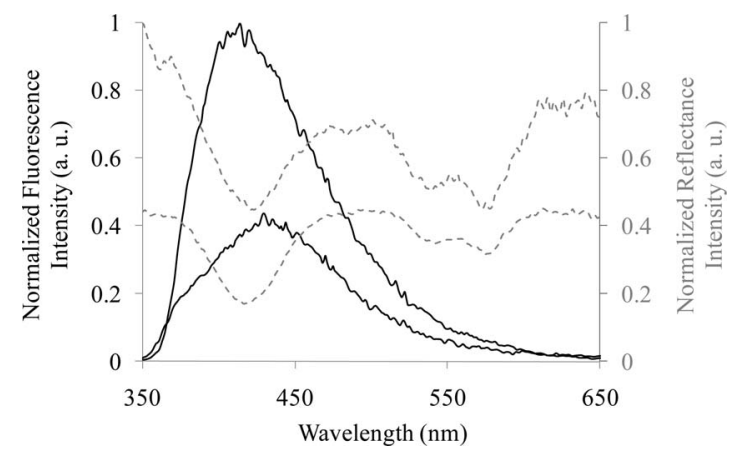

Fig. 3. Diffuse-reflectance (dashed lines) and fluorescence (solid lines) spectra examples. Higher intensity spectra correspond to normal tissue, whereas dysplastic tissue sites exhibit low-intensity spectra. For this illustration, reflectance and fluorescence intensity spectra were normalized to their maximum intensity values for a better comparison of their spectral shape (peaks and valleys).

and $q$ is the interference order. For the designed filters (see Fig. 2), the mirrors are dielectric mirrors composed of a stack of $\mathrm{TiO}_{2}$ and $\mathrm{SiO}_{2}$ thin-films (materials with high and low refractive index in the visible spectrum, respectively) which offer good optical performance characteristics, high transmittance and low full-width half-maximum (FWHM), with low-absorption losses [12], [22].

\section{B. Optical Filters Simulations}

To design the optical microsensors, i.e., to define the two specific transmittance spectral bands of the optical filters, diffusereflectance and fluorescence data from normal and dysplastic GI tissue were used (examples are shown in Fig. 3). This dataset comprised nine dysplastic tissue sites and 42 normal tissue sites.

From Fig. 3, and using only two spectral bands, the optical filters should be able to select one fluorescence band in the violet/blue region of the spectrum (centered at $420 \mathrm{~nm}$ ) and one reflectance band in the green region of the spectrum (centered at $540 \mathrm{~nm}$ ). These central wavelengths were selected in order to detect approximately the maximum fluorescence emission of tissues, and to detect one of the hemoglobin absorption peaks in the reflectance signal. Previous studies [23] have shown that it is very important to take into account a green reflectance band, to help in the differentiation of inflammation from tumors, and thus reduce the number of false positives. For this reason, the $540 \mathrm{~nm}$ spectral band was selected for the reflectance measurements, instead of the $420 \mathrm{~nm}$ spectral band.

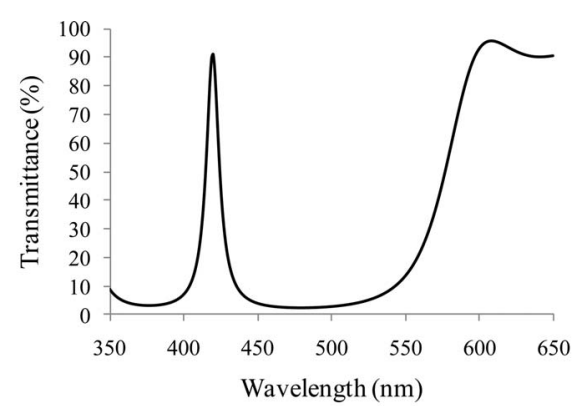

(a)

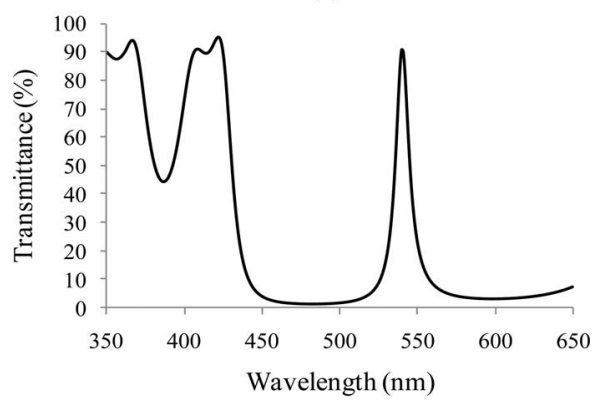

(b)

Fig. 4. Simulated spectral transmittance of the two Fabry-Perot optical filters: (a) violet/blue region; (b) green region. Maximum intensity peaks at (a) 420 and (b) $540 \mathrm{~nm}$.

For the violet/blue region, the mirrors are composed by $45 \mathrm{~nm}$-thick $\mathrm{TiO}_{2}$ and $70 \mathrm{~nm}$-thick $\mathrm{SiO}_{2}$ layers, whereas for the green region, the mirrors are composed by $52 \mathrm{~nm}$-thick $\mathrm{TiO}_{2}$ and $95 \mathrm{~nm}$-thick $\mathrm{SiO}_{2}$ layers. The resonance cavity thickness, i.e., the sixth layer, is equal to 138 and $192 \mathrm{~nm}$ for the violet/blue and green filter, respectively. In each spectral region, the optical filters can be easily tuned to a different central wavelength by adjusting only the thickness of the sixth layer, and keeping the same mirror layers. Thus, it would be possible to produce a filter centered at $450 \mathrm{~nm}$ by simply increasing the cavity thickness from 138 to $180 \mathrm{~nm}$, or to create a filter centered at $500 \mathrm{~nm}$ by reducing only the cavity thickness from 192 to $155 \mathrm{~nm}$. This procedure enables the fabrication of several optical filters in each region of the spectrum, whereas, minimizing the global deposition time.

Thin-film optics software TFCalc 3.5 (Software Spectra, Inc., Portland, OR) was used for the structural optimization of the optical filters. In Fig. 4, the simulated transmittances for the two Fabry-Perot optical channels are presented.

The simulated results show that each filter is sensitive to its specific spectral band, with FWHM less than $12 \mathrm{~nm}$. Also, the peaks transmittance is higher than $90 \%$. However, the tails of both filters at wavelengths farther from its transmittance peak may raise some concerns. For the violet/blue optical filter, the transmittance increases from 550 to $650 \mathrm{~nm}$. Given that this optical filter is going to be used solely for fluorescence measurements, this collection of out-of-band light is not a problem once fluorescence emission in this band tends to zero (see Fig. 3). On the other hand, as it is designed, the green optical filter will be collecting significant out-of-band light that will confound the reflectance analysis. Still, this problem can also be exceeded 


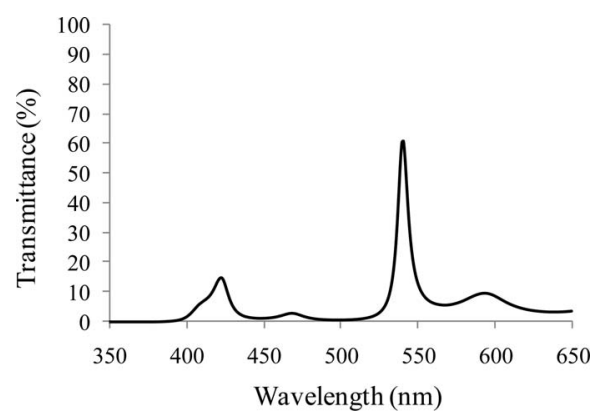

Fig. 5. Simulated spectral transmittance of the green filter with the optical effect of the $n+/ p$-epilayer photodiode included.

when the effect of the silicon photodiode is integrated in the measurements, together with the green filter, to produce the final device.

The integration of optical filters, photodiodes, and readout electronics on a single chip requires the system to fit in a microelectronic process, preferably CMOS. This technology features electronics in a small silicon area and offers low power consumption, an important requirement for the reported application. For the violet/blue spectral band, a photodiode with improved quantum efficiency at low-visible wavelengths is being designed. Regarding the green spectral band, we will use p-n junction photodiodes fabricated in a standard $0.7-\mu \mathrm{m}$ CMOS process, without additional masks or steps.

The CMOS process provides three photosensitive structures as vertical junction photodiodes. At a particular wavelength, their quantum efficiency varies according to their junction depth. In the visible spectrum, blue light is more efficiently collected by a shallower junction ( $p+/ n$-well and $n+/ p$-epilayer $)$ and red light by a deeper junction (n-well/p-epilayer). However, despite its shallow junction, the $n+/ p$-epilayer photodiode has high quantum efficiency around $550 \mathrm{~nm}$ due to the different doping concentration between the $n$ - and the $p$-side. This extends the $p$-side depletion area more deeply [24]. Therefore, this photodiode was selected for the microsystem. The green optical filter will be vertically aligned with the photodiode to build the optical channel for the collection of reflectance data. A detailed characterization of the fabricated $n+/ p$-epilayer photodiode can be found in [24].

New computational simulations were then performed for the green optical filter taking into account the effect of using an $n+/ p$-epilayer photodiode to read the signal. The thickness of the photodiode junction depth is fixed by the microelectronics foundry, and equals to $350 \mathrm{~nm}$. Fig. 5 illustrates the signal attenuation in the violet/blue region to less than $20 \%$. The peak transmittance is also lower than what was initially simulated due to the optical effect of the detector.

\section{RESUltS AND DisCUSSION}

\section{A. Thin-Film Optical Filters Characterization}

Before the optical filters fabrication, single layers of $\mathrm{SiO}_{2}$ and $\mathrm{TiO}_{2}$ were deposited on silicon substrates to calibrate deposition rates and indexes of refraction. After, the multilayer filters were

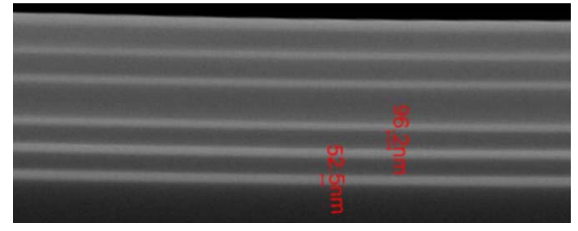

Fig. 6. SEM photograph showing the cross section of the green Fabry-Perot optical filter (magnification 100000 times).

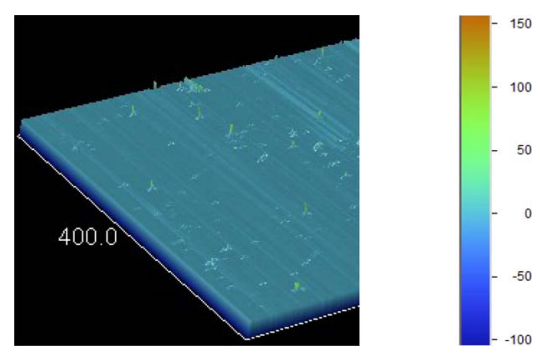

Fig. 7. Surface 3-D map of the green Fabry-Perot optical filter.

deposited by ion-beam deposition on a glass substrate using a Nordiko 3000 deposition tool. Multilayer filters were analyzed by a cross-sectional scanning electron microscope (SEM) in order to assess layer thicknesses and interface quality between the different layers (see Fig. 6).

In Fig. 6, it is noticeable the separation between the $\mathrm{SiO}_{2}$ and $\mathrm{TiO}_{2}$ layers, with very low interface mixing (brighter layers correspond to elements with high atomic number- $-\mathrm{TiO}_{2}$ ). The film thicknesses are in good agreement with the outlined values. It is also shown very good film flatness along the entire area. This feature allows us to guarantee the parallelism of the mirrors concerning to the resonance cavity, which is crucial for reproducible optical measurements.

The multilayer surface roughness was also evaluated, in a 400 by $400 \mu \mathrm{m}$ area, using a profilometer (Dektak 150 Surface Profiler, Veeco Instruments, Inc., Plainview, TX). The surface 3-D map for the green optical filter is shown in Fig. 7.

From Fig. 7, it can be seen that, in general, the optical filter's surface is smooth, as would be desirable in order to minimize potential interferences to the incident light beam. Its roughness profile is in average equal to $2.31 \mathrm{~nm}$. Several tiny irregularities inherent to the filters manufacturing process are present in the surface; yet, these are not a major concern due to their small size.

Finally, the transmittance performance of the optical filters was assessed (see Fig. 8). Optical spectra measurements on the filters show that they are sensitive to their particular spectral band. However, the violet/blue filter peak is slightly deviated from what was initially simulated, being centered at $422 \mathrm{~nm}$, which is explained by the fabrication process tolerance. This process has a tolerance of $2 \%$ in the thickness of the thin-film layers being deposited, which is more evident in the $\mathrm{SiO}_{2}$ : some $\mathrm{SiO}_{2}$ layers might have been deposited about 1 or $2 \mathrm{~nm}$ thicker than what was initially designed; an increase in the layers thickness shifts the spectral peak to the right. Still, this is a very small deviation that does not affect the performance of the overall 


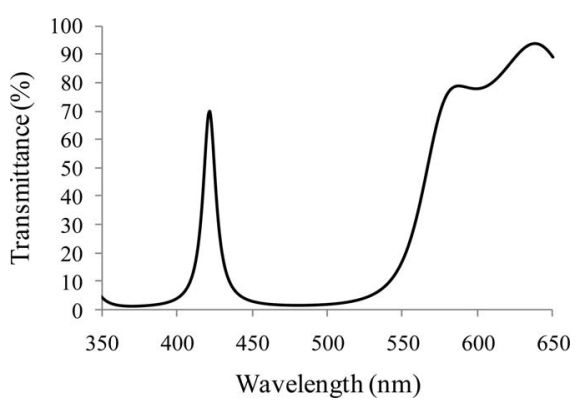

(a)

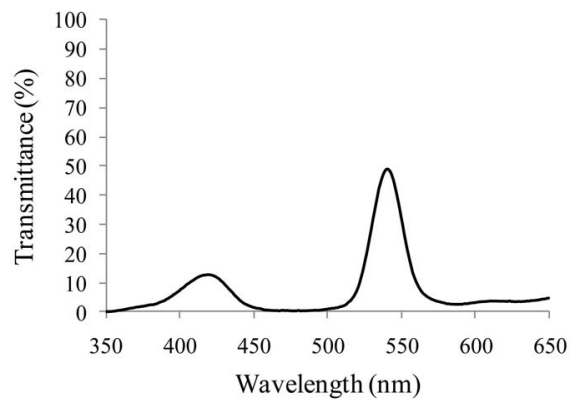

(b)

Fig. 8. Measured transmittance spectra of the two Fabry-Perot optical filters: (a) violet/blue filter, measured with the UV-3101 PC spectrophotometer (a CMOS photodiode is currently being designed and optimized for this spectral region); (b) green filter (measured with the fabricated $n+/ p$-epilayer photodiode). Maximum intensity peaks at (a) 422 and (b) $540 \mathrm{~nm}$

system. This filter spectral shape is in good agreement with the simulations, with a maximum transmittance around $70 \%$ and an FWHM of $12 \mathrm{~nm}$. The FWHM of the green filter is a little wider than expected by computational simulations (around $23 \mathrm{~nm}$ ). Its ratio of maximum transmittance to background noise is greater than 50/15, which is acceptable for the interference filter since the transmittance peak has more than twice the intensity of any background noise that appears in the considered spectral range.

The overall performance of the optical filters could be improved by increasing the number of dielectric layers, but the fabrication process complexity would also increase. Therefore, it must be achieved a compromise between the filters accuracy to perform diagnosis and the constraints inherent to the filters fabrication process (e.g., materials, number of layers, FWHM, and maximum transmission).

\section{B. Diffuse-Reflectance and Fluorescence Spectra Analysis}

A new strategy based on the use of only two spectral bands for the detection of dysplasia in the upper GI tract was studied. The feasibility of using the developed optical microsensors for distinguishing dysplasia from normal tissue was theoretically assessed with a spectral analysis. For this study, the optical filters transmittance curves were used together with spectroscopy diffuse-reflectance and fluorescence data. Histopathology diagnoses are used as the standard against which the spectroscopicqualitative diagnoses are compared.

In this analysis, all diffuse-reflectance and fluorescence spectra (not normalized) were first multiplied by the green and violet/blue filters measured transmittance curves, respectively (see
Fig. 8). The resulting Gaussian distributed spectra were then integrated over the full transmittance range (from 350 to $650 \mathrm{~nm}$ ). The obtained integrated intensity values correspond to the signal that will be read by the photodiodes.

Subsequently, a statistical analysis was performed on the processed spectral data using the Student's $t$-test, the MannWhitney test, and the logistic regression model. This analysis is performed in order to extract any relevant diagnostic algorithm that provides diagnostic differences between normal and dysplastic tissues. A statistical software $\left(\operatorname{IBM}^{\circledR}\right.$ SPSS $\left.^{\circledR}\right)$ was used for this study.

The Student's $t$-test and the Mann-Whitney test, respectively, were used to compare normally and not normally distributed continuous variables (diffuse-reflectance intensity in the green spectral band and fluorescence intensity in the violet/blue spectral band) for two distinct groups of tissue sites: dysplastic and normal. A $p$-value less than 0.05 was considered to be statistically significant. For both variables, the null hypothesis was rejected. This means that the measured intensity values of diffuse-reflectance at $540 \mathrm{~nm}$, and of fluorescence at $420 \mathrm{~nm}$, are significantly different between the two groups. In nearly all cases, compared with dysplasia, normal tissue sites exhibit significantly higher intensity values, both for diffuse-reflectance and fluorescence, as it was theoretical expected. As explained in Section II, a decrease in the fraction of collagen fibers with progression of dysplasia is translated by low fluorescence intensities, and by a reduced reflectance signal as a result of low scattering. Also, a high hemoglobin concentration, which is characteristic of dysplastic tissues, may attenuate the diffuse-reflectance and endogenous fluorescence signals due to absorption.

The spectral algorithm for classifying tissue as normal or dysplastic was developed by using binary logistic regression. A model with a good predictive capability includes both the diffuse-reflectance and fluorescence data. The accuracy of the final logistic regression model is evaluated by the area under the receiver operating characteristic (ROC) curve (AUC), and the sensitivity and specificity. The box plots of the reflectance and fluorescence variables, as well as the ROC curve for the differentiation of dysplasia from normal tissue are presented in Fig. 9.

From the results of Fig. 9, one can say that the violet/blue fluorescence and green diffuse-reflectance intensities are good predictors for the discrimination of tissue disease status. The model has an overall accuracy of $94.1 \%$. Dysplasia and normal tissue can be distinguished with an AUC, sensitivity, and specificity of $0.995,77.8 \%$, and $97.6 \%$, respectively. Sensitivity is defined as the fraction of dysplastic tissue sites correctly identified by the model, whereas specificity is defined as the fraction of correctly identified normal tissue sites. The not very high value of sensitivity might be explained by the small number of dysplastic tissue sites used, which is a limitation in this study. The positive predictive value, which is the probability that a positive result is accurate equals to $87.5 \%$. The negative predictive value (NPV), which is the probability that a negative result is accurate, is equal to $95.3 \%$. A high NPV (low number of false negatives) is very important for an effective clinical 


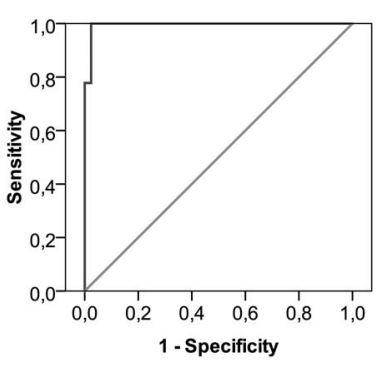

(a)

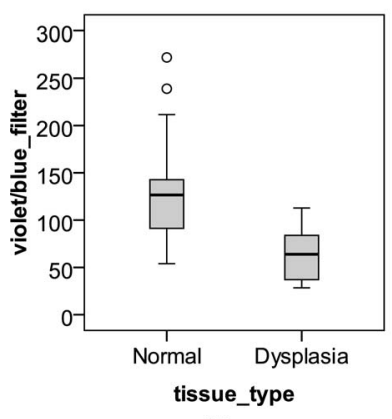

(b)

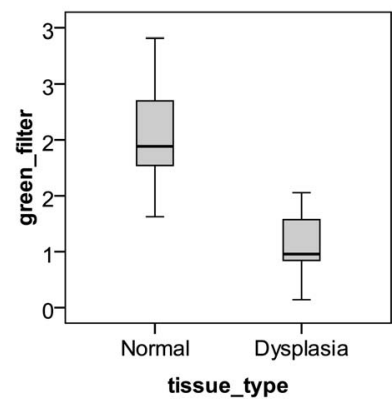

(c)
Fig. 9. Discrimination of normal from dysplastic tissue sites. (a) ROC curve for the logistic regression algorithm: ROC curve (blue solid line); $45^{\circ}$ green line $(\mathrm{AUC}=0.5)$. The AUC measures the accuracy of the model to correctly classify normal and diseased tissue. A perfect discrimination is achieved with an AUC value of 1 ; an AUC value of 0.5 indicates an inability to differentiate the two tissue types. Box plots of the two variables included in the model. (b) Fluorescence data from the violet/blue spectral region for the two tissue types. (c) Reflectance data from the green spectral region for the two tissue types. Outliers are symbolized by circles.

practice, especially when the system is going to be used to guide biopsies, since the physician has to be confident that none of the dysplastic lesions are identified as normal, and consequently not subjected to biopsy. A low NPV might be indicative that cancerous tissue is left in the patient without being identified.

Mayinger et al. [18] described a measurement system that uses differences in the fluorescence spectral intensities to identify cancerous tissue in the upper GI tract. Tissues were excited with violet-blue light and fluorescence spectra were collected using an optical fiber and analyzed with a spectrograph. The developed algorithm was based on the intensity ratio in the green and red spectral bands and provided a sensitivity of $97 \%$ and a specificity of $95 \%$ for the diagnosis of esophageal cancer. However, in this study, it was not included data for benign lesions (e.g., inflammations) that might influence fluorescence spectroscopy. Uedo et al. [23] found that a superficial neoplasm and an inflamed tissue with edema could be misunderstood using only fluorescence measurements. In this study, the addition of green reflectance information proved to be helpful for the differentiation of inflammation from tumors.

Bourg-Heckly et al. [6] also used a fluorescence spectroscopy system for the detection of dysplasia in the upper GI tract. The instrument was composed by a UV xenon lamp, bandpass filters, optical fibers for light delivery and collection, and a spectrometer with a CCD camera for detection. Overall, this optical setup is not portable and compact, which might be cumbersome in the clinical environment. In this study, a model based only on the flu- orescence intensity ratio at two different wavelengths (390 and $550 \mathrm{~nm}$ ) was able to discriminate neoplastic tissue from normal mucosa with a sensitivity of $86 \%$ and specificity of $95 \%$.

In our study, we not only combine information from diffusereflectance and fluorescence, which combination was shown to be helpful for the discrimination of benign and malignant lesions, but we also demonstrate that it is possible to identify dysplastic and healthy tissue sites, with a suitable sensitivity and specificity, using a much smaller instrument, compared with the instruments used in similar studies. Replacing the spectrograph by thin-film optical filters and the CCDs by silicon photodiodes will enable the development of a more portable, low cost, and simple spectroscopy system, with similar throughput.

The obtained results with only two optical filters are very promising but further study should be done. As an important next step, the performance of the presented diagnostic algorithm should be tested in a large clinical study using the optical microsensor incorporated in a conventional endoscope. This optical microsensor must have not only the optical filters postprocessed directly on top of the CMOS photodiodes but also the readout electronics, all bonded together on the same chip. A separated fabrication was used as a first approach, since it enabled the characterization and optimization of the different parts.

\section{CONCLUSION}

Diffuse-reflectance and fluorescence spectroscopy proved to be sensitive methods for the detection of early cancerous lesions in the GI tract. This study reports a new approach for the identification of dysplasia using a simple and miniaturized system for spectroscopy data collection. The proposed device is based on thin-film optical filters and silicon photodiodes for the selection and detection of two spectral bands that will provide information for diagnosis.

An empirical study was performed using spectroscopy data from dysplastic and normal GI tissue sites to demonstrate the feasibility of replacing the spectrograph in standard clinical prototypes by two optical filters in the miniaturized system. The spectral performance of the fabricated interference filters was shown to be sufficient for the intended final goal. The overall results have proven that healthy and diseased tissue can be differentiated with a sensitivity and specificity of $77.8 \%$ and $97.6 \%$, respectively.

It is reasonable to envision an important clinical utility for the proposed optical microsensor since due its reduced size it could be incorporated in a standard endoscope making it more compact and portable. Moreover, it could also be, in the future, integrated in an endoscopic capsule platform for guiding biopsy of invisible lesions in the GI tract. With this device, the sampling error and healthcare costs associated with unnecessary biopsies could be significantly reduced.

\section{ACKNOWLEDGMENT}

The authors would like to thank Prof. A. C. Braga for the helpful discussions in statistics. 


\section{REFERENCES}

[1] J. Ferlay, H. R. Shin, F. Bray, D. Forman, C. Mathers, and D. M. Parkin. (2010). GLOBOCAN 2008, Cancer Incidence and Mortality Worldwide: IARC CancerBase No. 10. Lyon, France: Int. Agency Res. Cancer. [Online]. Available at http://globocan.iarc.fr/.

[2] J. W. Tunnell, A. E. Desjardins, L. Galindo, I. Georgakoudi, S. A. McGee, J. Mirkovic, M. G. Mueller, J. Nazemi, F. T. Nguyen, A. Wax, Q. Zhang, R. R. Dasari, and M. S. Feld, "Instrumentation for multi-modal spectroscopic diagnosis of epithelial dysplasia," Technol. Cancer Res. Treat., vol. 2, pp. 505-514, 2003.

[3] C. C. Yu, C. Lau, G. O'Donoghue, J. Mirkovic, S. McGee, L. Galindo, A. Elackattu, E. Stier, G. Grillone, K. Badizadegan, R. R. Dasari, and M. S. Feld, "Quantitative spectroscopic imaging for non-invasive early cancer detection," Opt. Exp., vol. 16, pp. 16227-16239, 2008.

[4] I. Georgakoudi, "The color of cancer," J. Lumin., vol. 119-120, pp. 75-83, 2006.

[5] B. Mayinger, P. Horner, M. Jordan, C. Gerlach, T. Horbach, W. Hohenberger, and E. G. Hahn, "Light-induced autofluorescence spectroscopy for tissue diagnosis of GI lesions," Gastrointestinal Endoscopy, vol. 52, pp. $395-400,2000$.

[6] G. Bourg-Heckly, J. Blais, J. J. Padilla, O. Bourdon, J. Etienne, F. Guillemin, and L. Lafay, "Endoscopic ultraviolet-induced autofluorescence spectroscopy of the esophagus: Tissue characterization and potential for early cancer diagnosis," Endoscopy, vol. 32, pp. 756-765, 2000.

[7] B. Mayinger, M. Jordan, P. Horner, C. Gerlach, S. Muehldorfer, B. R. Bittorf, K. E. Matzel, W. Hohenberger, E. G. Hahn, and K. Guenther, "Endoscopic light-induced autofluorescence spectroscopy for the diagnosis of colorectal cancer and adenoma," J. Photochem. Photobiol. B: Biol., vol. 70, pp. 13-20, 2003.

[8] J. Y. Lo, B. Yu, H. L. Fu, J. E. Bender, G. M. Palmer, T. F. Kuech, and N. Ramanujam, "A strategy for quantitative spectral imaging of tissue absorption and scattering using light emitting diodes and photodiodes," Opt. Exp., vol. 17, pp. 1372-1384, 2009.

[9] M. Kfouri, O. Marinov, P. Quevedo, N. Faramarzpour, S. Shirani, L. W. C. Liu, Q. Fang, and M. J. Deen, "Toward a miniaturized wireless fluorescence-based diagnostic imaging system," IEEE J. Sel. Topics Quantum Electron., vol. 14, no. 1, pp. 226-234, Jan./Feb. 2008.

[10] A. Piegari and J. Bulir, "Thin film filters with variable transmittance for spectrometry," in Proc. 4th IEEE/LEOS Workshop Fibres Opt. Passive Compon., Jun. 2005, pp. 109-144.

[11] D. P. Poenar and R. F. Wolffenbuttel, "Optical properties of thin-film silicon-compatible materials," Appl. Opt., vol. 36, pp. 5122-5127, 1997.

[12] H. A. Macleod, Thin-Film Optical Filters, 3rd ed. London, U.K.: Inst. Phys. Publishing, 2001.

[13] G. Zonios, L. T. Perelman, V. Backman, R. Manoharan, M. Fitzmaurice, J. Van Dam, and M. S. Feld, "Diffuse reflectance spectroscopy of human adenomatous colon polyps in vivo," Appl. Opt., vol. 38, pp. 6628-6637, 1999.

[14] I. Georgakoudi, B. C. Jacobson, M. G. Müller, E. E. Sheets, K. Badizadegan, D. L. Carr-Locke, C. P. Crum, C. W. Boone, R. R. Dasari, J. Van Dam, and M. S. Feld, "NAD $(\mathrm{P}) \mathrm{H}$ and Collagen as in vivo quantitative fluorescent biomarkers of epithelial precancerous changes," Cancer Res., vol. 62, pp. 682-687, 2002.

[15] A. Couvelard, F. Paraf, V. Gratio, J. Y. Scoazec, D. Hénin, C. Degott, and J. F. Fléjou, "Angiogenesis in the neoplastic sequence of Barrett's oesophagus. Correlation with VEGF expression," The J. Pathology, vol. 192, pp. $14-18,2000$.

[16] N. Ramanujam, "Fluorescence spectroscopy of neoplastic and nonneoplastic tissues," Neoplasia, vol. 2, pp. 89-117, 2000

[17] R. S. Dacosta, B. C. Wilson, and N. E. Marcon, "Spectroscopy and fluorescence in esophageal diseases," Best Pract. Res. Clin. Gastroenterol., vol. 20, pp. 41-57, 2006

[18] B. Mayinger, P. Horner, M. Jordan, C. Gerlach, T. Horbach, W. Hohenberger, and E. G. Hahn, "Light-induced autofluorescence spectroscopy for the endoscopic detection of esophageal cancer," Gastrointestinal Endoscopy, vol. 54, pp. 195-201, 2001.

[19] D. C. De Veld, M. J. Wities, H. J. Sterenborg, and J. L. Roodenburg, "The status of in vivo autofluorescence spectroscopy and imaging for oral oncology," Oral Oncol., vol. 41, pp. 117-131, 2005.

[20] A. Menciassi, A. Moglia, S. Gorini, G. Pernorio, C. Stefanini, and P. Dario, "Shape memory alloy clamping devices of a capsule for monitoring tasks in the gastrointestinal tract," J. Micromech. Microeng., vol. 15, pp. 20452055,2005
[21] M. Quirini, S. Scapellato, P. Valdastri, A. Menciassi, and P. Dario, "An approach to capsular endoscopy with active motion," in Proc. IEEE Eng. Med. Biol. Soc. Conf., 2007, pp. 2827-2830.

[22] G. Minas, R. F. Wolffenbuttel, and J. H. Correia, "An array of highly selective Fabry-Perot optical-channels for biological fluid analysis by optical absorption using a white-light source for illumination," J. Opt. A: Pure Appl. Opt., vol. 8, pp. 272-278, 2006.

[23] N. Uedo, H. Iishi, M. Tatsuta, and T. Yamada, "A novel videoendoscopy system by using autofluorescence and reflectance imaging for diagnosis of esophagogastric cancers," Gastrointestinal Endoscopy, vol. 62, pp. 521$528,2005$.

[24] G. Minas, J. C. Ribeiro, R. F. Wolffenbuttel, and J. H. Correia, "On-chip integrated CMOS optical detection microsystem for spectrophotometric analyses in biological microfluidic systems," in Proc. IEEE Int. Symp Ind. Electron., Jun. 2005, pp. 1133-1138.

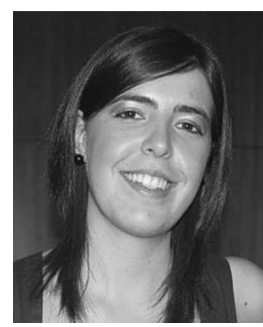

D. S. Ferreira (S'09) received the M.Sc. degree in biomedical Eegineering (major in medical electronics) from the University of Minho, Guimarães, Portugal, in 2007, where she has been working toward the Ph.D. degree in the Department of Industrial Electronics, under the MITPortugal Program, since October 2007.

Her research interests include spectroscopy for tissue diagnosis, biomedical microdevices, and optical microsensors.

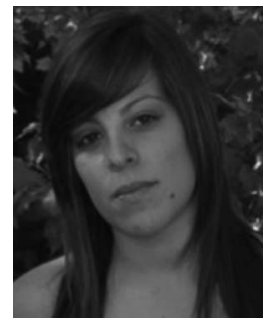

V. C. Pinto is currently working toward the M.Sc. degree in micro/nano Technologies in the Department of Industrial Electronics, University of Minho, Guimarães, Portugal.

Her research interests include microfabrication techniques for microfluidic devices and thin-film characterization.

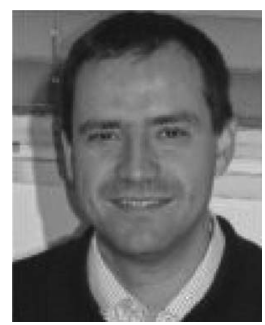

J. H. Correia (S'96-M'00) received the B.Sc. degree in physical engineering from the University of Coimbra, Coimbra, Portugal, in 1990, and the Ph.D. degree at the Laboratory for Electronic Instrumentation, Delft University of Technology, The Netherlands, in 1999, working in the field of microsystems for optical spectral analysis.

$\mathrm{He}$ is currently a Full Professor in the Department of Industrial Electronics, University of Minho, Guimarães, Portugal. He was the General-Chairman of Eurosensors 2003 and MME 2007, Guimarães. His research interests include micromachining and microfabrication technology for mixed-mode systems, solid-state integrated sensors, microactuators, and microsystems.

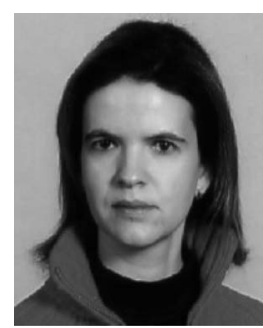

G. Minas (S'96-M'05-SM'11) received the B.Sc. degree in industrial electronics engineering in 1994 , the M.Sc. degree in 1998, and the Ph.D. degree in 2004, all from the University of Minho, Guimarães, Portugal.

Her thesis work was in cooperation with the Laboratory for Electronic Instrumentation, Delft University of Technology, Delft, The Netherlands, and dealt with lab on a chips for biological fluids analysis. Between 1995 and 2004, she was a Lecturer, and from 2004 to 2010, an Assistant Professor in the Department of Industrial Electronics, University of Minho, where she has been an Associate Professor since 2010, where she is involved in biomedical microdevices research, in general, and on lab-on-a-chip devices with on-chip integration of electronic circuits, optical filters, sensors, biosensors, and microactuators. 